\title{
Ultrastructural basis for different pertechnetate uptake patterns by various human brain tumours
}

\author{
P. B A R-S E L LA, D. FR ONT, R. HAR D OFF, E. PEYSER, \\ B. BOR OVICH, A N D I. NIR
}

From the Unit of Electron Microscopy, the Division of Nuclear Medicine, and the Department of Neurosurgery, Rambam Medical Center and the Technion-Faculty of Medicine, Haifa, Israel

S UMMARY Patterns of pertechnetate uptake were correlated with ultrastructural properties of the endothelial wall in 14 human brain tumours. In tumours with reduced uptake of the radionuclide, intercellular tight junctions were observed whereas absence of intercellular tight junctions was characteristic of all tumours with an increased uptake of pertechnetate. In some tumours with increased uptake, fenestrated endothelial wall was seen while in others nonfenestrated wall was evident. We concluded that intercellular junctions and not fenestrations affect the permeability of brain tumours to pertechnetate.

The uptake of ${ }^{99^{\mathrm{m}}} \mathrm{Tc}$ pertechnetate by tumour tissue is the basis for the visualisation of brain tumours by scintigraphy. Various patterns of pertechnetate uptake have been found in brain scintigraphy. Some tumours are visualised early and intensely, others late or not at all (Penning and Front, 1975). Two main factors govern the passage of Tc-pertechnetate from the blood into the tissue of the tumours - the vascularity of the tumour, and the permeability of the blood-brain barrier.

In a previous study, uptake of pertechnetate was found to be independent of vascularity (Front, 1978). It was suggested that changes in the bloodbrain barrier, which is localised in the capillary wall, might be involved with the different rates of ${ }^{99 \mathrm{~m}} \mathrm{Tc}$-pertechnetate uptake. In the present study we extended our investigations to include the ultrastructural properties of the capillary wall. Thus, both scintigraphic assessments and electron microscopic studies were carried out on the same tumour, in order to correlate given ultrastructural features with scintigraphic patterns of pertechnetate uptake.

\section{Subjects and methods}

Fourteen patients whose clinical condition enabled detailed scintigraphic assessments and whose removed tumours were adequately processed for

Address for correspondence and reprint requests: Professor D. Front, Division of Nuclear Medicine, Rambam Medical Center, Haifa, Israel. Accepted 30 March 1979 electron microscopy, were included. Radionuclide angiography was performed by a gamma camera after injection of $15 \mathrm{mCi}$ of radioactive technetium pertechnetate $\left({ }^{99 \mathrm{~m}} \mathrm{Tc}\right)$. An early four-view study collecting 400000 counts per view followed. A static study was done after two hours. The same technique was used for ${ }^{99^{\mathrm{m}} \mathrm{Tc}} \mathrm{T}$-labelled red blood cells ( ${ }^{99^{\mathrm{m}}}$ Tc RBC) (Front, 1978).

Samples of brain tumours were taken from the patients during surgery and placed immediately in cold glutaraldehyde $2.5 \%$ in phosphate buffer $(0.1 \mathrm{M} ; \mathrm{pH} 7.2)$. After five minutes in the fixative the tissue was cut into small blocks and left in fresh fixative for two hours at $4^{\circ} \mathrm{C}$. The specimens were washed in the same buffer and post-fixed in osmium tetroxide $1 \%$ for one hour. After dehydration with ethanol the tissue was embedded in Epon 812. Thin sections were stained with uranyl acetate and lead citrate, and examined in the EM JEOL operating at $80 \mathrm{kV}$.

\section{Results}

The permeability of the tumour demonstrated by early and late pertechnetate uptake, vascularity demonstrated by ${ }^{99^{\mathrm{m}}} \mathrm{Tc}$-labelled red blood cells, and the ultrastructural features of the various tumours are shown in the Table. Tumours were considered to have high permeability when they were apparent in radionuclide angiography and early static study, and were significantly more prominent in the pertechnetate study than in the 
Table Scintigraphic assessments and ultrastructural features of brain tumours

\begin{tabular}{|c|c|c|c|c|c|c|}
\hline \multirow[t]{2}{*}{ Case } & \multirow[t]{2}{*}{ Histological diagnosis } & \multicolumn{2}{|c|}{ Scintigraphic assessments } & \multicolumn{2}{|c|}{ Ultrastructural features } & \\
\hline & & Permeability & Vascularisation & Fenestrations & Tight junctions & \\
\hline 1 & Meningioma & +++ & +++ & + & - & \\
\hline 2 & Meningioma & +++ & +++ & + & - & \\
\hline 3 & Meningioma & +++ & +++ & + & - & 9 \\
\hline 4 & Choroid plexus papilloma & +++ & +++ & + & - & $\overline{7}$ \\
\hline 5 & Glioblastoma multiforme & ++ & +++ & + & - & $>$ \\
\hline 6 & Metastatic carcinoma & ++ & not available & + & - & \\
\hline 7 & Metastatic carcinoma & +++ & $0-+$ & + & - & \\
\hline 8 & Astrocytoma grade 2 & +++ & + & - & - & \\
\hline 9 & Glioblastoma multiforme & +++ & + & - & - & 8 \\
\hline 10 & Glioblastoma multiforme & ++ & ++ & - & - & 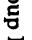 \\
\hline 11 & Glioblastoma multiforme & +++ & ++ & - & - & $\varpi$ \\
\hline 12 & Medulloblastoma & +- & $0-+$ & - & + & $c$ \\
\hline 13 & Glioblastoma multiforme & +- & not available & - & + & 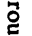 \\
\hline 14 & Glioblastoma multiforme & +- & $0-+$ & - & + & 0 \\
\hline
\end{tabular}

${ }^{99^{\mathrm{m}}} \mathrm{Tc}$-labelled red blood cells study. Vascularity was assessed by the appearance on scintigraphy with Tc-labelled red blood cells. High permeability and vascularity were marked $(+++)$, medium $(++)$, and low $(+)$. When the tumour was not visualised at all, it scored (0).

In the electron microscopy analysis, only capillaries and post-capillary venules were included. Attention was given primarily to structural characteristics of the endothelial wall which might affect the uptake of pertechnetate. Thus, the presence of intercellular tight junctions and the presence of fenestrations in the endothelial wall were described (Table). The presence of pinocytotic vesicles was not included in Table, but all the observed tumours contained numerous vesicles, including those with low uptake of pertechnetate.

Three cases of meningioma, one case of glioblastoma multiforme, and one case of choroid plexus papilloma showed high pertechnetate permeability and high vascularity (Table, cases 1,2 , $3,4,5)$. In Fig. 1a-d the scintigrams and representative micrographs taken from a meningioma are shown. This group of tumours (Table, group A) demonstrated fenestrated type endothelial wall with open intercellular junctions. The fenestrations were closed with a thin diaphragm (Fig. 1c). In the thicker non-fenestrated part of the cytoplasm, filaments, and pinocytotic vesicles were observed (Fig. 1).

Most of the junctions between adjacent endothelial cells were long and somewhat convoluted and were difficult to observe at a favourable plan. However, when a shorter junction was sectioned at a right angle an open gap was clearly visualised (Fig. 1d).
In two cases of metastatic carcinoma, quick and intense uptake of pertechnetate was seen (Table, cases 6 and 7, group A). This was coupled in one patient (case 7) with low vascularity. The ultrastructural characteristics were similar to those found in meningiomas.

In one case of astrocytoma grade 2 and three cases of glioblastoma multiforme, medium to high pertechnetate permeability was found (Table, cases 8-11, group B). In astrocytoma (Fig. 2b) and one case of glioblastoma (cases 8 and 9) low vascularity was observed, while in the two other cases of glioblastoma (cases 10 and 11) medium vascularity was recorded.

Ultrastructurally, this group of tumours (Table, group B) revealed non-fenestrated type endothelial wall together with open intercellular junctions. In the astrocytoma, about half of the tumour capillaries consisted of highly hypertrophic endothelial cells (Fig. 2c). The thicker part of the cell bulged into the lumen and almost obliterated it. The uptake of pertechnetate was obviously not affected by these structural abnormalities as could be seen from the high permeability shown on the scintigrams (Fig. 2a).

In Fig. $2 d$ the full length of an open intercellular junction is shown. The same ultrastructural features, the absence of fenestrations together with the absence of tight junctions, were observed also in the three glioblastomas (cases 9, 10, 11) of group $B$.

In two cases of glioblastoma and one case of medulloblastoma (Table, cases 12, 13, 14) low permeability was observed. This group of tumours (Table, group C) was the only one in which intercellular tight junctions between the endothelial cells were seen. 

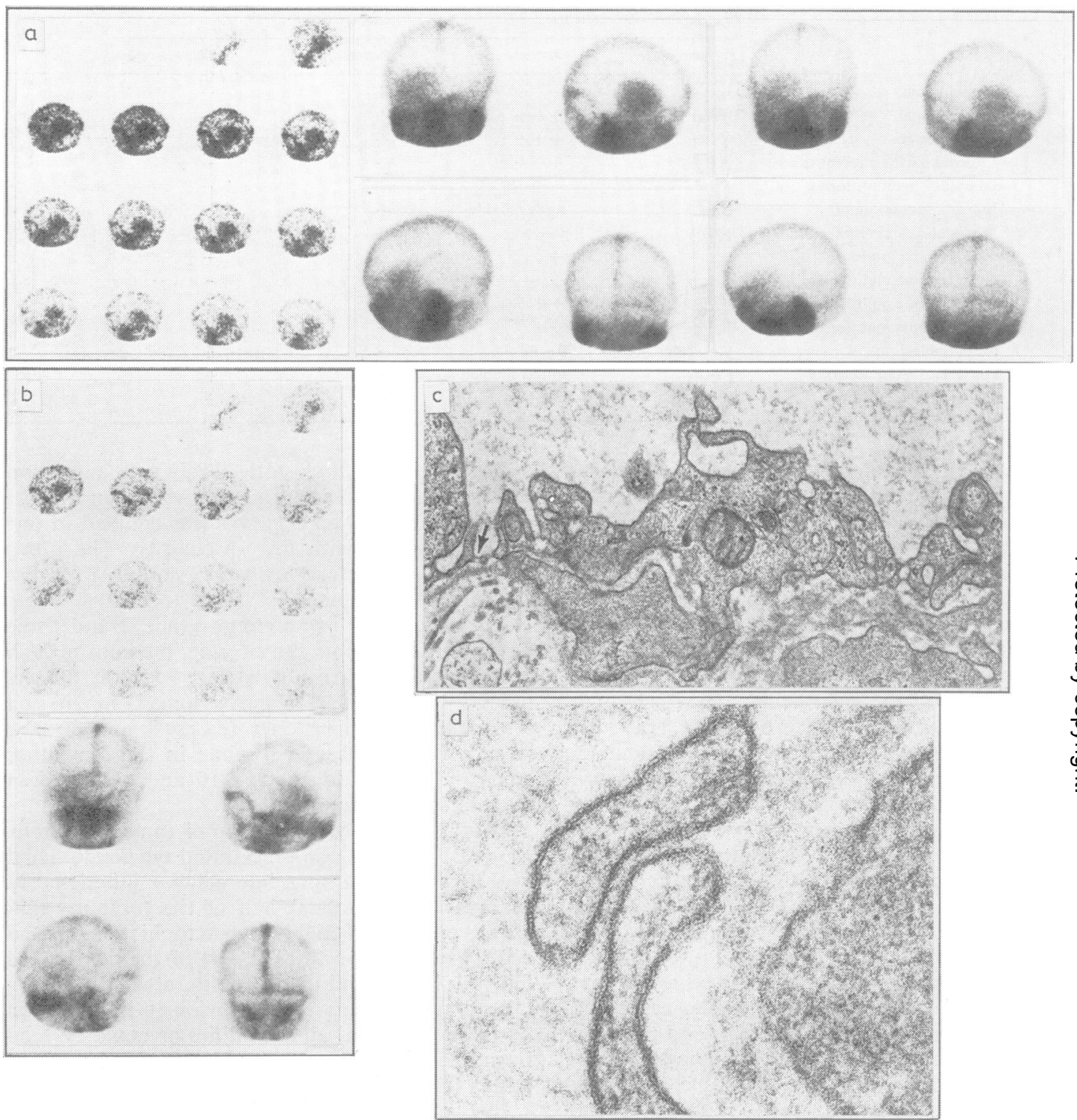

Fig. 1 Meningioma (case 1). (a) Increased permeability is shown by ${ }^{99 m}$ Tc pertechnetate study.

Radionuclide angiography (left), early static study (centre), and late static study (right). Early and marked visualisation of the right temporal tumour. (b) Rich vascularity is shown by ${ }^{9{ }^{9} m}$ Tc-labelled red blood cells. Radionuclide angiography (top) and static study (bottom). Marked and early visualisation decreasing in intensity in dynamic study. Marked vascularity in static study. (c) Fenestrations in the capillary endothelial wall, closed with diaphragms (arrow). A number of pinocytotic vesicles and microfilaments are seen in the endothelial cytoplasm. Original magnification $\times 25000$. (d) An open gap between adjacent endothelial cells. Original magnification $\times 160000$. 

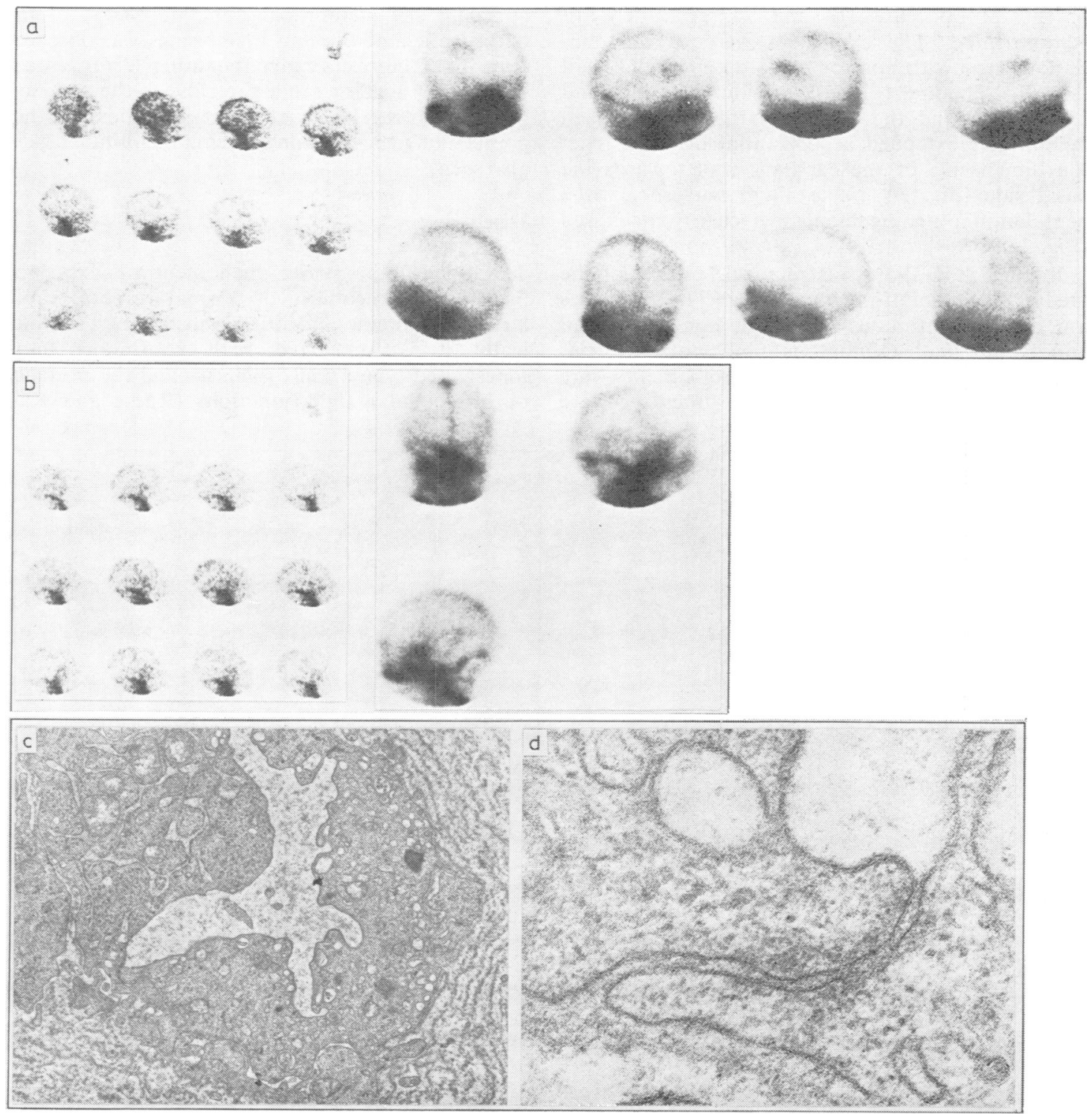

Fig. 2 Astrocytoma grade 2 (case 8). (a) Increased permeability is shown by ${ }^{99 m}$ Tc pertechnetate study. Radionuclide angiography (left), early static study (centre), and late static study (right). The lesion is shown early and persists throughout the study. (b) Low vascularity is shown by ${ }^{99 m}$ Tc-labelled red blood cells. Radionuclide angiography (left) and static study (right). The lesion is barely visible (c) Continuous type endothelial wall is seen. Hypertrophic endothelial cells are surrounding an almost occluded lumen. The blood vessel is encircled by a thick, multilayered basal membrane. Numerous pinocytotic vesicles are seen in the endothelial cytoplasm. Original magnification $\times 10000$. (d) The full length of an open intercellular junction is visualised at high magnification. Original magnification $\times 120000$. 
In Fig. 3a-c the scintigraphic assessment and the ultrastructural features of the glioblastomas of this group (cases 13 and 14, group C) are demonstrated. The capillaries were of the continuous type with non-fenestrated endothelial wall (Fig. 3b). The intercellular junctions were long and convoluted, and the fusion of the opposing cell membranes extended a substantial distance from the lumen side of the capillary wall towards the basal side (Fig. 3b, c). At high magnification a pentalaminar arrangement was clearly visualised (Fig. 3c).

In Fig. 4a, b the ultrastructural features of the medulloblastoma are demonstrated. The capillaries are of the continuous type with non-fenestrated endothelial wall. Numerous pinocytotic vesicles and relatively high content of microfilaments were observed (Fig. 4a). Intracellular junctions were relatively short and the full length of the junction could be resolved. At different points along the opposing cell membranes punctate fusion of the outer leaflets of the unit membranes was observed (Fig. 4b). The resulting pentalaminar arrangement was usually localised in a site close to the capillary lumen (arrow). However, tight junctions at other points along the opposing plasma membranes were also seen

\section{Discussion}

The blood-brain barrier phenomenon is expressed by the impermeability of normal cerebral capillaries to proteins and other substances. Anatomically it is associated with continuous nonfenestrated type brain capillaries and the presence of intercellular tight junctions (Reese and Kar-
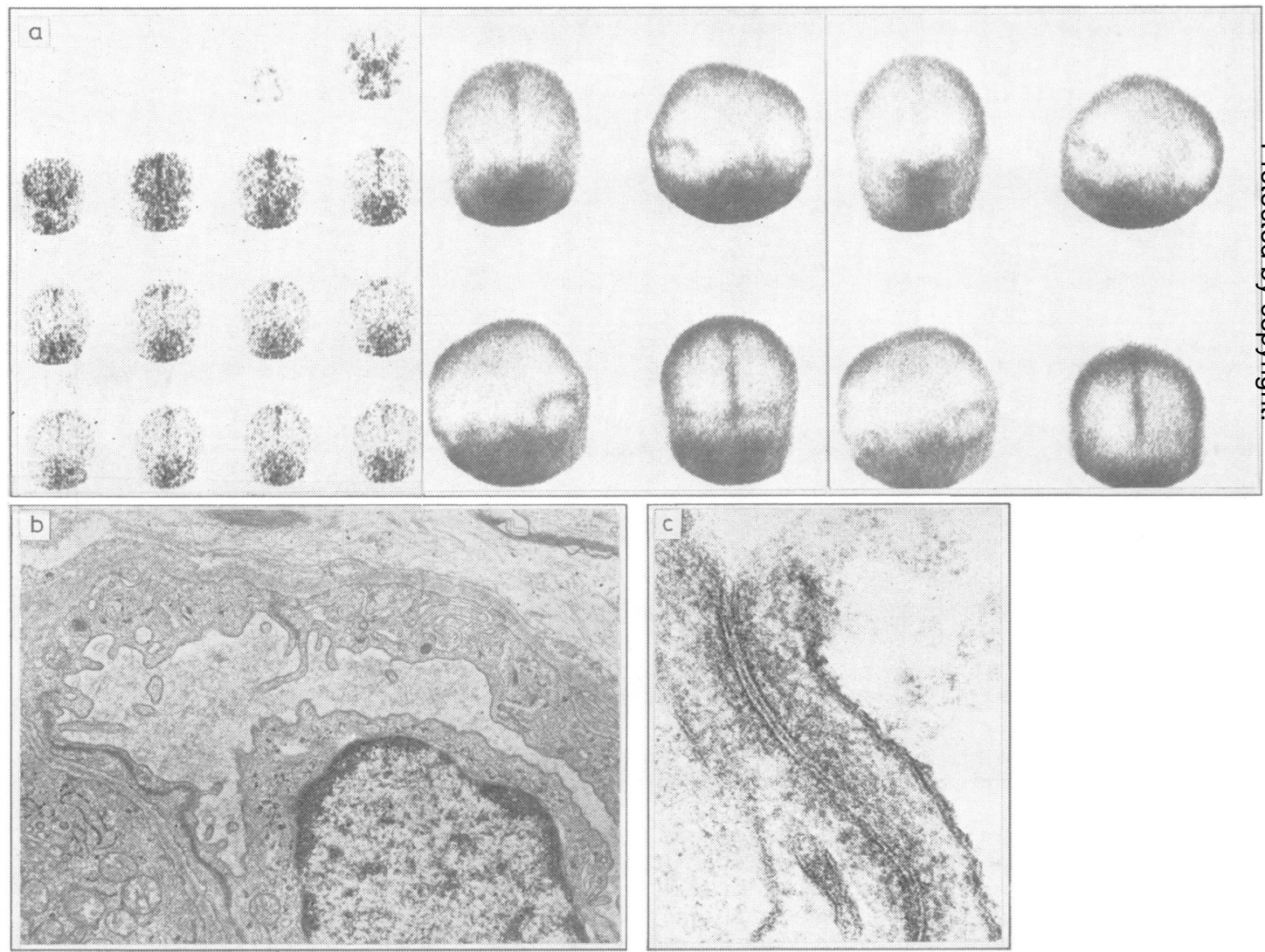

Fig. 3 Glioblastoma multiforme (case 13). (a) Low permeability is shown by ${ }^{99 m}$ Tc pertechnetate study. Radionuclide angiography (left), early static (centre), and late static study (right). Some vague poorly defined uptake is seen in the right hemisphere. (b) Continuous type endothelial wall and intercellular tight junctions. Original magnification $\times 13500$. (c) High magnification of opposing cell membranes. Fusion of the outer leaflets of adjoining unit membranes. Original magnification $\times 160000$. 


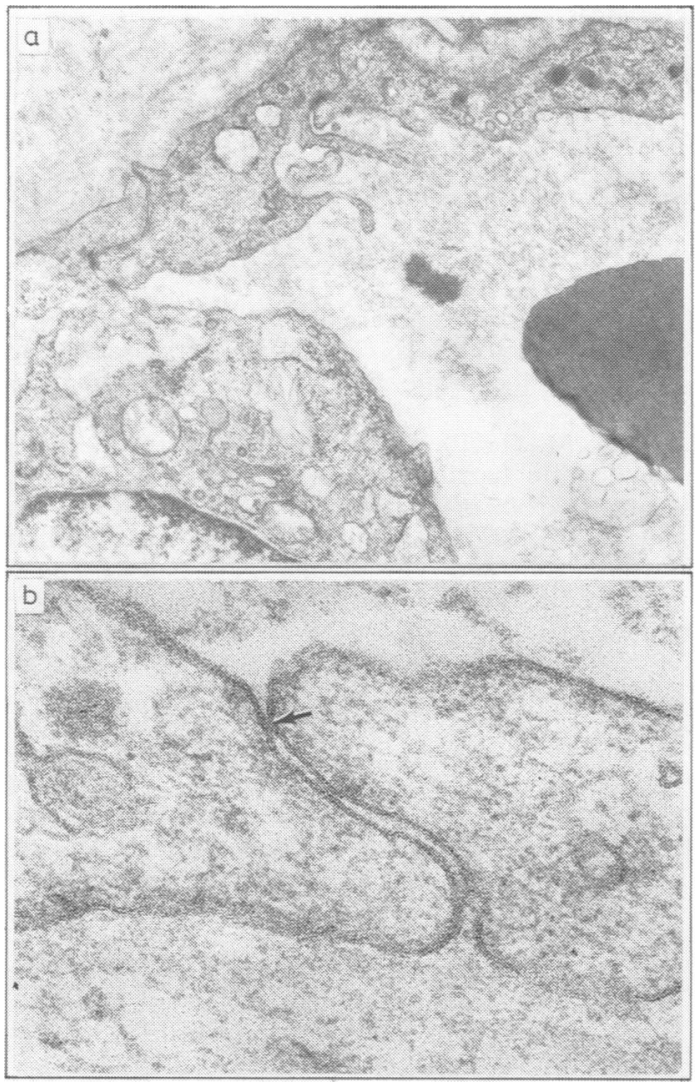

Fig. 4 Medulloblastoma (case 12). (a) Continuous type endothelial wall is demonstrated. The endothelial cytoplasm contains numerous microfilaments and pinocytotic vesicles. Part of a red blood cell is seen in the lumen. Original magnification $\times 20000$. (b) The full length of a short unconvoluted junction is visualised. Contact between opposing cell membranes and the formation of a pentalaminar arrangement, typical of a tight junction (arrow) is seen at the lumen side. Original magnification $\times 140000$.

novsky, 1967; Brightman et al., 1970). It was suggested that the tight junctions between endothelial cells are of the zonula occludens type, forming a complete belt around the cells (Reese and Karnovsky, 1967). This arrangement prevents the passage of horseradish peroxidase, lanthanum, and other test substances from the blood into the brain (Reese and Karnovsky, 1967). Pertechnetate is also excluded from brain tissue by the normal CNS vessels but penetrates into brain tumours.

In the present study we have attempted to correlate the various patterns of pertechnetate uptake-that is, rapid and intense uptake, inter- mediate slow uptake, and almost no uptake-in certain tumours, with their capillary ultrastructure. In previous studies, changes in the bloodbrain barrier in human tumours were suggested (Brightman et al., 1970) but given structural features were not correlated with an in vivo penetration of a test substance.

In glioblastomas the absence of the blood-brain barrier was demonstrated by the use of fluoresceinprotein tracers. It was correlated with open endothelial cell junctions and fenestrated capillary walls (Long, 1970). In haemangioblastoma (Tani et al., 1974) metastatic renal carcinoma (Hirano and Zimmerman, 1972), and Schwannoma (Long, 1973), the increased permeability of the capillaries was explained by the presence of fenestrations in the endothelial cells, open junctions, and perhaps numerous pinocytotic vesicles.

The ultrastructure of the capillaries of all the common types of meningiomas was examined by Long (1973). These tumours were permeable to protein-bound tracers. Endothelial junctions were patent as was demonstrated by lanthanum nitrate. The tracer was found throughout the open junctions of the endothelial cells.

Our results showed that fenestrated type endothelial wall was found in a number of brain tumour capillaries, but the presence of fenestrations could not be correlated with the degree of pertechnetate uptake. Thus, medium to high uptake of pertechnetate was found not only in tumours with fenestrated capillaries (Table, group A) but also in tumours with non-fenestrated capillaries (Table, group B). It seems, therefore, that fenestrations cannot be regarded as the main structural substrate which determines pertechnetate uptake by tumours.

The absence of intercellular tight junctions, on the other hand, was a persistent common feature of all tumours in which scintigraphic assessments showed an increased uptake of pertechnetate. Whenever open junctions were observed, medium to high pertechnetate accumulation was observed, regardless of the extent of vascularity or the presence of fenestrations. Moreover, in the three cases with reduced pertechnetate uptake, a medulloblastoma and two glioblastomas, tight junctions were observed. Although these tumours had nonfenestrated endothelial walls, it is reasonable to conclude, based on the other cases, that it is the presence of tight junctions which determined the low uptake of pertechnetate.

Pinocytotic vesicles were seen in all the tumours tested. As transendothelial passage through this route would be too slow to account for the rapid entry of pertechnetate into the tumour, no effort 
was made to measure the number of pinocytotic vesicles. Moreover, as different tumours with high permeability contain variable numbers of pinocytotic vesicles, a significant correlation between pinocytotic vesicles and the rate of pertechnetate uptake could not be drawn.

The correlation of pertechnetate uptake as determined by scintigraphy, with the absence of intercellular tight junctions might be important in planning cancer chemotherapy in the individual patient (Vick et al., 1977). Brain tumours with predetermined elevated uptake of pertechnetate might be accessible to chemotherapy via the open route of intercellular junctions. On the other hand, in cases which show no significant uptake of pertechnetate, chemotherapy would not be effective, as the pharmaceutical would be excluded from the tumour by the intercellular tight junction in the endothelial wall. It should be noted, however, that variations in the width of open junctions were observed. The gap between adjacent endothelial cells varied from less than $75 \AA$ in the astrocytoma to $200 \AA$ and more in meningiomas $(1 \AA=10 \mathrm{~mm})$. This variation, although only moderately affecting the uptake of pertechnetate, might be of greater significance if larger organic molecules are applied.

Studies on animals are now in progress to correlate the uptake of labelled drugs used in chemotherapy with the ultrastructural features of endothelial junctions in brain tumours.

\section{References}

Brightman, M. W., Klatzo, I., Olsson, I., and Reese, T. S. (1970). The blood-brain barrier to proteins under normal and pathological conditions. Journal of the Neurological Sciences, 10, 215-239.

Front, D. (1978). Scintigraphic assessment of vascularity and blood-tissue barrier of human brain tumours. Journal of Neurology, Neurosurgery, and Psychiatry, 41, 18-23.

Hirano, A., and Zimmerman, H. M. (1972). Fenestrated blood vessels in a metastatic renal carcinoma in the brain. Laboratory Investigation, 26, 465-468.

Long, D. M. (1970). Capillary ultrastructure and the blood brain barrier in human malignant brain tumors. Journal of Neurosurgery, 32, 127-144.

Long, D. M. (1973). Vascular ultrastructure in human meningiomas and schwannomas. Journal of Neurosurgery, 38, 409-419.

Penning, L., and Front, D. (1975). Brain Scintigraphy. A Neuroradiological A pproach, pp. 43-49. Excerpta Medica: Amsterdam, New York.

Reese, T. S., and Karnovsky, M. J. (1967). Fine structural localization of a blood-brain barrier to exogenous peroxidase. Journal of Cell Biology, 34, 207-217.

Tani, E., Ikeda, K., Kudo, S., Yamagata, S., Higashi, N., and Fujihara, E. (1974). Fenestrated vessels in human hemangioblastoma. Journal of Neurosurgery $\frac{2}{\infty}$ 40, 696-705.

Vick, N. A., Khandekar, J. D., and Ringer, D. D. (1977). Chemotherapy of brain tumors. The bloodo brain barrier is not a factor. Archives of Neurology (Chicago), 34, 523-526. 